\section{Estudio imagenológico del síndrome aórtico agudo}

\author{
CRISTIÁN VARELA ${ }^{1}$, ENZO MANTELLI ${ }^{a}$, PATRICIA RÍOS ${ }^{a}$, \\ JOSÉ M. ZÚÑIGA ${ }^{\text {, }}$ MARÍA J. MEZA ${ }^{\mathrm{b}}$, CAROLINA FUCHS $^{\mathrm{b}}$, \\ VERUSKA DE LUCCAS ${ }^{1}$, JOSÉ MIGUEL GUTIÉRREZ ${ }^{1}$
}

\section{Imaging approach to acute aortic syndrome}

Acute aortic syndromes include a spectrum of life-threatening aortic conditions. A review of the diagnostic aspects of the acute aortic syndrome was made, from the perspective of the imaging techniques available for this purpose. The advantages and disadvantages of each technique and its diagnostic performance were evaluated. Emphasis was placed on the relevance of clinical information as a fundamental tool for suspecting this syndrome and appropriately choosing the imaging technique. Our main objective is to provide information about the diagnosis of this condition, especially in the context of emergency services.

(Rev Med Chile 2019; 147: 1579-1593)

Key words: Acute Disease; Chest Pain; Aneurysm, Dessecting; Aortic Aneurysm; Hematoma, Subdural.
'Unidad de Imágenes de Cuerpo, Departamento de Imagenología, Clínica Dávila, Santiago, Chile aMédico general, Research Fellow Investigación, Departamento de Imagenología, Clínica Dávila. Santiago, Chile.

${ }^{\text {b} A l u m n o ~ d e ~ M e d i c i n a ~(a y u d a n t e ~}$ alumno), Universidad de los Andes. Santiago, Chile.

Trabajo no recibió financiamiento Los autores declaran no tener conflictos de interés.

Recibido el 31 de enero de 2019, aceptado el 16 de octubre de 2019.

Correspondencia a:

Dr. Cristián Varela Ubilla Av. Recoleta 464, Recoleta, Santiago, Chile. cvarela@davila.cl
$\mathrm{E}$ 1 síndrome aórtico agudo (SAA) es el término utilizado para describir un conjunto de patologías de la aorta cuyo síntoma cardinal es el dolor torácico agudo, las cuales pueden poner en riesgo la vida del paciente, por lo que su reconocimiento y manejo adecuado es fundamental para la sobrevida.

Las condiciones que conforman el SAA, determinan diferentes formas de disrupción de la arquitectura normal de la pared aórtica. Cabe recordar que esta tiene 3 capas $^{1}: 1$ ) Interna o íntima, 2) Media y 3) Externa o adventicia (Figura 1). Los procesos de mayor prevalencia provocan una modificación anatómica en una pared enferma o afectada por procesos degenerativos (ateromatosis). Es menos frecuente que existan factores de origen congénito de la pared que predispongan a estos procesos. Finalmente estos procesos pueden ocurrir en una pared aórtica sana en el contexto de trauma o iatrogenia.

\section{Tipos de condiciones incluidas en el síndrome aórtico agudo (SAA) ${ }^{2}$}

Disección aórtica (DA) aguda (Figura 2.A)

Separación de las capas de la pared aórtica por irrupción de sangre a través de un desgarro de la íntima.

- Espontánea: Rotura de la íntima con disección secundaria de la media o por hemorragia de la media, que subsecuentemente se comunica al lumen a través de un defecto de la íntima. En ambos casos se crea un lumen verdadero y uno falso. La disección puede extenderse distal o proximalmente a la lesión inicial, pudiendo comprometer: válvula aórtica, ostium de las arterias coronarias, ramas de la porción torácica o abdominal lo que determinará manifestaciones clínicas y complicaciones ${ }^{3}$.

- Iatrogénica o traumática: Secundaria a lesión intimal por balón de contrapulsación aórtico, procedimiento endovascular o bien durante un accidente de alta energía, por ejemplo en vehículo motorizado.

Hematoma intramural (HIM) aórtico (Figura 2.B)

Se debe a un sangrado en la media, secundaria a rotura y/o hemorragia de los vasos nutricios (vasa vasorum) de la pared aórtica (Figura 3). Corresponde a un hematoma confinado a la capa media en ausencia de desgarro detectable de la íntima, sin embargo, pueden existir microdesgarros. 


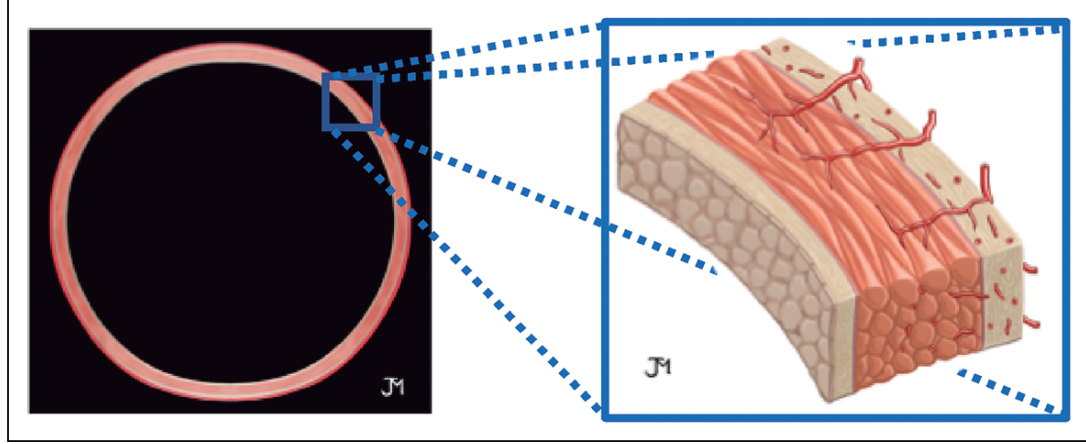

Figura 1. Anatomía aorta normal, con amplificación de pared donde se observa vasa vasorum en capa media.
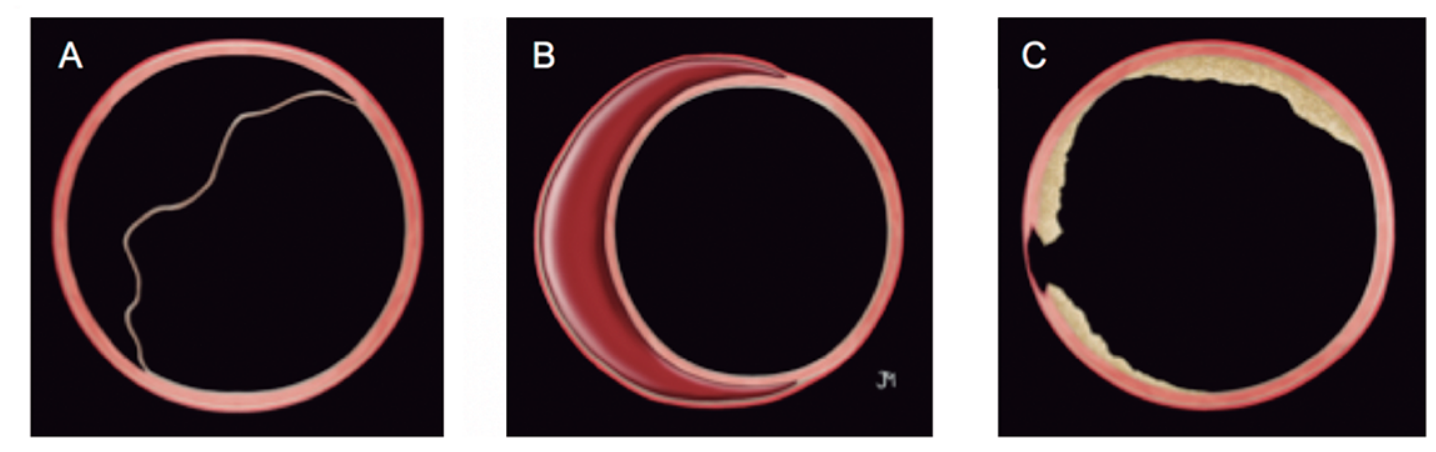

Figura 2. A. Disección aórtica; B. Hematoma intramural; C. Úlcera aórtica penetrante.

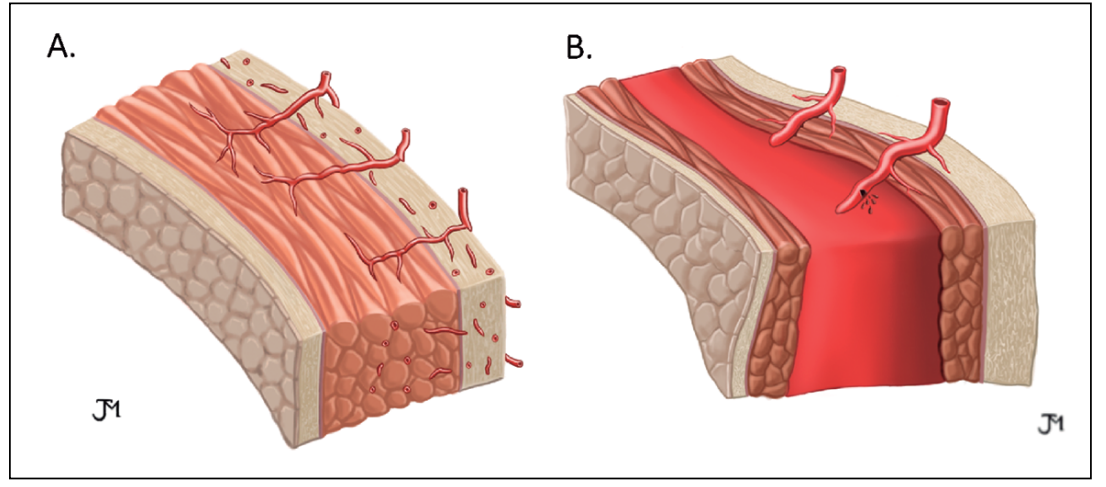

Figura 3. Esquema representativo de pared aórtica. A. Pared aórtica normal. B. Formación de hematoma intramural.

Puede ser precursor de la DA aguda en $8-12 \%$ de los $\operatorname{casos}^{4,5}$. Su etiología puede ser espontánea o iatrogénica/traumática.

\section{Úlcera aórtica penetrante (UAP) (Figura 2.C)}

Ulceración de una lesión arteriosclerótica de la pared aórtica que penetra en la lámina elástica interna, formando un hematoma en la capa media, con o sin trombo subyacente ${ }^{6}$. Es precursora de DA en $<5 \%$ de los casos.

\section{Otras variantes recientemente incluidas en el $S A A$}

1. Desgarro de la intima sin hematoma: Variable poco común de la DA, se caracteriza por desgarro lineal o estrellado de la íntima con 
exposición de la capa media o adventicia ${ }^{7}$, sin separación de las capas. Una lesión traumática no penetrante puede manifestarse de esta manera.

2. Hematoma periaórtico: Representa una ruptura aórtica contenida con sangrado periaórtico. El 23\% de las DA cursan con un hematoma periaórtico ${ }^{8}$. Implicando mayores tasas de shock, taponamiento cardíaco, alteraciones de conciencia/coma y mortalidad, que aquellas que se presentan sin este.

\section{Clasificación anatómica del SAA}

Las lesiones que constituyen el SAA se clasifican de acuerdo a su localización y extensión. Para la DA existen 2 clasificaciones, que también sirven para describir al HIM:

- Clasificación de Stanford: Es la más utilizada. Las lesiones que comprometen a la aorta ascendente se clasifican tipo A, independiente del sitio de la lesión primaria. Si no involucra la aorta ascendete será de tipo $\mathrm{B}^{3}$ (Figura 4 ).

- Clasificación de DeBakey: Se basa en el origen de la lesión. Tipo I: se origina en aorta ascendente y extendiéndose al menos hasta el arco aórtico. Tipo II: se origina en la aorta ascendente y está confinado a ella. Tipo III: se origina en la aorta descendente y puede extenderse proximal o distalmente?
Las DA torácicas que afectan la porción ascendente son casi el doble de las que comprometen la porción descendente. El sitio más comúnmente afectado es la pared lateral derecha de la aorta ascendente. Los HIM afectan comúnmente la porción descendente. La mayoría de las UAP se localizan en la porción descendente $(85-95 \%)^{3}$.

\section{Epidemiología y factores de riesgo ${ }^{3}$}

- La incidencia del SAA es de 2 a 4 casos por 100.000 individuos.

- DA aguda: es la más común. Sus factores de riesgo son: hipertensión arterial $(77,8 \%)$, ateroesclerosis $(31 \%)$, cirugía cardiaca o torácica previa, aneurisma conocido, patologías del tejido conectivo y válvula aórtica bicúspide.

- Es más común en hombres $(65 \%$ de los casos), y la presentación en mujeres es más tardía.

- El factor precipitante más importantes es la hipertensión sistémica, primaria o secundaria.

- HIM: 5-20\% de los casos de SAA. Comparado con la DA, suelen ser pacientes mayores, y presentar aneurismas. Además muy pocos presentan Síndrome de Marfan y es más prevalente en mujeres. Al igual que en la disección el factor de riesgo más importante es la hipertensión arterial de larga data (50-84\% de los casos).

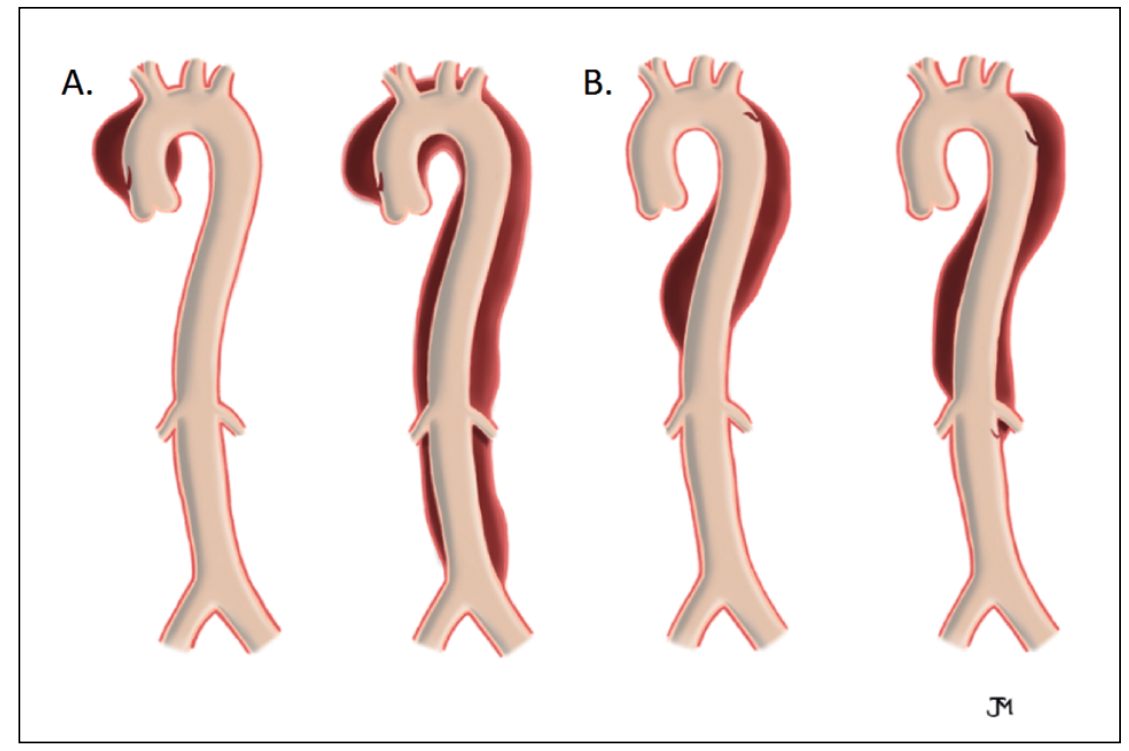

Figura 4. Clasificación de Standford. A. Disección aórtica tipo A. B. Disección aórtica tipo B. 
- UAP: 2-7\% de los pacientes con SAA. Suelen ser mayores de 70 años. Los factores de riesgo asociados son: ateroesclerosis, hipertensión, hiperlipidemia, enfermedad coronaria, tabaquismo, aneurisma aórtico infrarrenal.

\section{Manifestaciones clínicas de SAA}

\section{Cuadro clínico}

El dolor torácico especialmente de tipo transfixiante en un paciente mayor e hipertenso debe despertar la sospecha de SAA. Es frecuente la coexistencia de varios factores de riesgo (Tabla 1), los que junto a las características del cuadro clínico (Tabla 2) sugieren este síndrome. Las entidades que conforman el SAA pueden solaparse o confundirse clínicamente, de manera que para confirmar la sospecha y diferenciarlas entre cada una, se deben realizar exámenes de imágenes de alto rendimiento diagnóstico, que permitan además demostrar su extensión e identificar las complicaciones.

Tabla 1. Patologías asociadas (factores de riesgo) en pacientes portadores de DA torácica tipo B, según $R$. Mertens et al. ${ }^{10}$

\begin{tabular}{|lc|}
\hline Hipertensión arterial & $77,8 \%$ \\
\hline Tabaquismo & $38,9 \%$ \\
\hline Dislipidemia & $25,0 \%$ \\
\hline Obesidad & $11,1 \%$ \\
\hline Insuficiencia renal crónica & $8,3 \%$ \\
\hline Enfermedad de Marfán & $8,3 \%$ \\
\hline Cardiopatía coronaria & $5,6 \%$ \\
\hline
\end{tabular}

SCORE clínico de riesgo de $\mathrm{DA}^{12,13}$

- Aortic Dissection Detection Risk Score (ADDRS) (Tabla 3 y 4): El uso de la ADD-RS junto al dímero $\mathrm{D}$, sería una eficiente combinación para el enfrentamiento inicial de estos pacientes.

A continuación, se propone un esquema de aproximación ante pacientes con sospecha de SAA, para determinar riesgo de este y eventual estudio a realizar (Figura 5).

\section{Exámenes de laboratorio}

\section{Dímero $D^{12}$}

Representa un producto de la degradación de fibrina, por lo que su rol en eventos trombóticos está bien establecido. Múltiples estudios han demostrado que este biomarcador también

\section{Tabla 2. Síntomas y signos en los pacientes} portadores de una DA aguda ${ }^{11}$

\begin{tabular}{|ll|}
\hline Dolor severo o el "peor de la vida" & $90 \%$ \\
\hline Inicio abrupto del dolor & $90 \%$ \\
\hline Dolor torácico con o sin irradiación al dorso & $85 \%$ \\
\hline Hipertensión (PAS $\geq 150 \mathrm{mmHg}$ ) & $49 \%$ \\
\hline Insuficiencia aórtica & $32 \%$ \\
\hline Dolor abdominal & $30 \%$ \\
\hline Pulso parvus et tardus & $27 \%$ \\
\hline Dolor migratorio & $19 \%$ \\
\hline $\begin{array}{l}\text { Hipotensión (PAS < } 100 \mathrm{mmHg}, \text { shock o } \\
\text { taponamiento cardíaco }\end{array}$ & $18 \%$ \\
\hline Déficit neurológico focal & $12 \%$ \\
\hline
\end{tabular}

PAS, presión arterial sistólica. Parvus et tardus, pulso disminuido en amplitud y de ascenso lento.

Tabla 3. SCORE clínico de riesgo de DA (ADD-RS)

\section{Dolor con características de alto Condición de alto riesgo riesgo \\ Torácico, Dorsal, o abdominal descrito como inicio súbito, intensidad severa, o como desgarrante \\ Síndrome de Marfán, historia familiar de enfermedad aórtica, antecedentes de enfermedades de válvula aórtica, manipulación reciente aórtica, aneuris- ma de aorta torácica conocido}

\section{Hallazgo al examen físico de alto riesgo}

Evidencia de déficit de perfusión (pulso débil y/o asimétrico, diferencia en Presión arterial en extremidades, o déficit neurológico focal con dolor), nuevo soplo aórtico de insuficiencia con dolor, hipotensión/shock 
Imágenes en síndrome aórtico agudo - C. Varela et al

\section{Tabla 4. Manejo según resultado}

Manejo según resultado

ADD-RS > 1: considerar realizar directamente angio TC u otra imagen concluyente

$A D D-R S \leq 1$ : Realizar dímero $D$, dímero $D<500 \mathrm{ng} / \mathrm{mL}$, considerar descartar SAA, con resultado $\geq 500 \mathrm{ng} / \mathrm{mL}$, considerar angio TC

ADD-RS: Aortic Dissection Detection Risk Score; TC: Tomografía computada; SAA: Síndrome aórtico agudo.

tiene una alta sensibilidad $(94,5 \%)$ en el SAA, pero en forma aislada, un resultado negativo es insuficiente para poder descartarlo. Fisiopatológicamente se cree que esta correlación es secundaria a la injuria tisular, que produciría una liberación del factor tisular el que activaría la cascada de coagulación.
ECG

Debe realizarse en todo paciente con sospecha de SAA, aunque en la gran mayoría de los pacientes se encontrará normal. Su gran aporte es descartar un Síndrome Coronario Agudo (SCA).

\section{Traslado a la Unidad de Imagenología}

En esta fase de la atención de los pacientes, eventuales portadores de una SAA, es de vital importancia, ya que pueden ocurrir eventos prevenibles. Dentro de la valoración inicial es fundamental tener disponibilidad de radiografía de tórax y ecografía portátil para descartar causas de dolor que deban resolverse en forma inmediata y puedan agravarse en el traslado a la Unidad de TC, como por ejemplo un neumotórax masivo. También debe lograrse un adecuado control del dolor para que el paciente pueda colaborar en la obtención de las imágenes. Una adecuada moni-

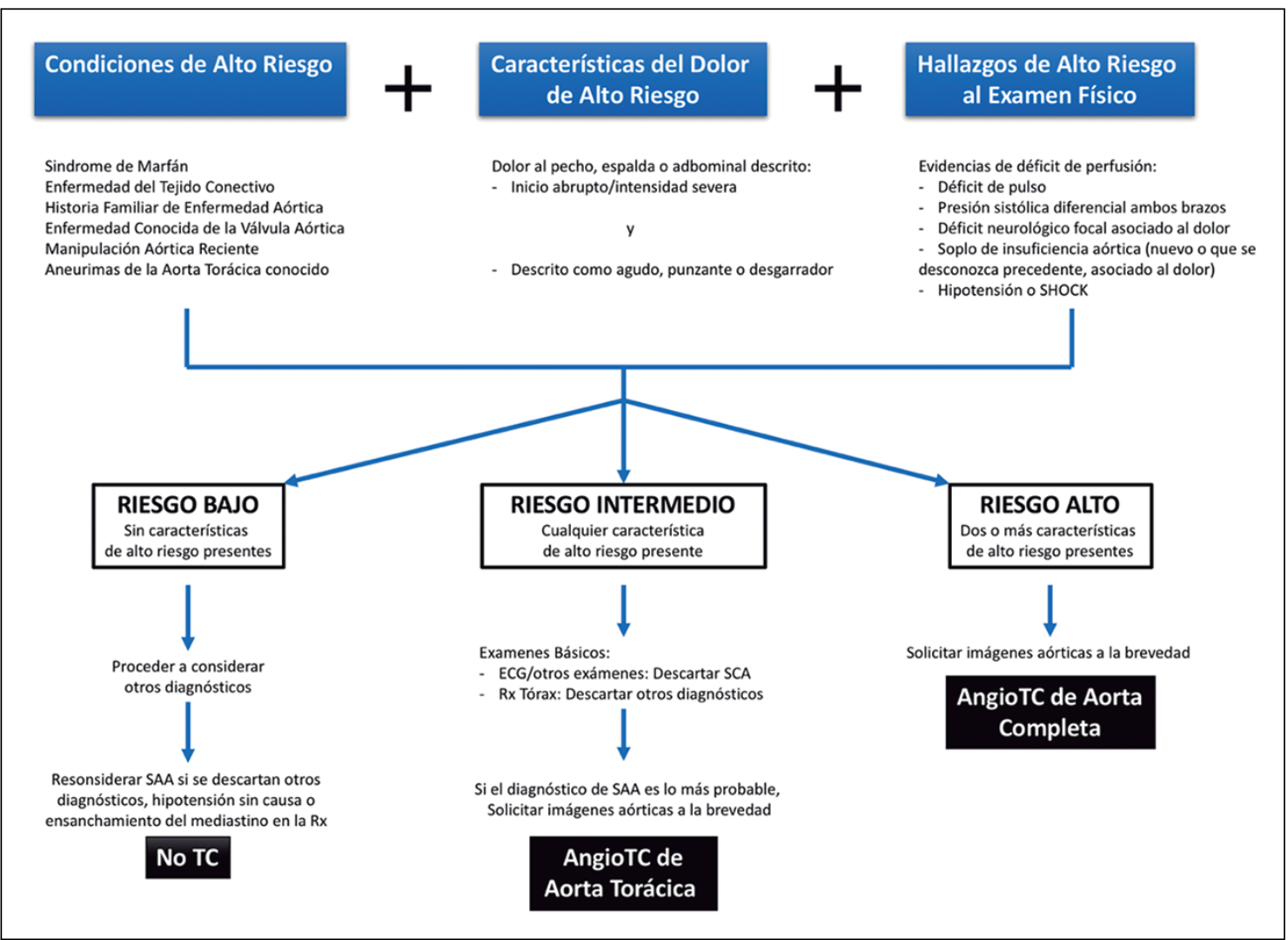

Figura 5. Esquema de estratificación de riesgo para SAA y estrategias de manejo apropiado, traducido y modificado a partir de Vardhanabhuti $\vee$ et al. ${ }^{14}$. 
torización de los signos vitales y supervisión por personal entrenado reducirá los riesgos durante el tránsito a la unidad de imágenes o hacia las unidades de pacientes críticos ${ }^{15,16}$.

\section{Estudios imagenológicos}

La sospecha clínica se confirma mediante exámenes de imagen. Existen múltiples exámenes imagenológicos en el estudio del SAA, todos tienen ventajas y desventajas, y la elección será determinada dependiendo del paciente y la disponibilidad local (Tabla 5). Actualmente, el Angio-TC, especialmente con técnica multicorte (MC), es el examen de elección para evaluar el SAA en el Servicio de Urgencias.

Tabla 5. Ventajas, desventajas y precaución de los distintos métodos imagenológicos para el diagnóstico del SAA ${ }^{17}$

\begin{tabular}{|c|c|c|c|}
\hline Examen & Ventajas & Desventajas & Precaución \\
\hline \multirow[t]{3}{*}{ Rx de Tórax } & Baja dosis de radiación & Baja especificidad & $\begin{array}{l}\text { Falso positivo en caso de } \\
\text { tortuosidad aórtica, tumor } \\
\text { mediastínico, derrame pleural } \\
\text { o pericárdico, insuficiencia } \\
\text { cardíaca }\end{array}$ \\
\hline & Realizable en Box de reanimación & $\begin{array}{l}\text { Baja sensibilidad en el diagnóstico } \\
\text { de enfermedades aórticas }\end{array}$ & \\
\hline & $\begin{array}{l}\text { Muy útil para descartar otras } \\
\text { patologías agudas (neumotórax o } \\
\text { derrame pleural) }\end{array}$ & $\begin{array}{l}\text { Bajo valor predictivo negativo } \\
\text { en enfermedades cardíacas o } \\
\text { pulmonares }\end{array}$ & \\
\hline \multirow[t]{7}{*}{ TCMC } & $\begin{array}{l}\text { Muy alta sensibilidad y especifici- } \\
\text { dad }(100 \%)\end{array}$ & Exposición a radiación & $\begin{array}{l}\text { Falsos positivos en SAA en } \\
\text { caso de: a) Alta densidad de la } \\
\text { pared aórtica; b) Artefacto por } \\
\text { movimiento de pared de la aor- } \\
\text { ta ascendente; c) Ateromatosis } \\
\text { significativa de la pared aórtica }\end{array}$ \\
\hline & $\begin{array}{l}\text { Requiere menos tiempo de ejecu- } \\
\text { ción que RM }\end{array}$ & $\begin{array}{l}\text { Potencial nefrotoxicidad y contra- } \\
\text { indicación relativa en pacientes } \\
\text { con antecedentes de reacción tipo } \\
\text { alérgica con medio de contraste } \\
\text { yodado }\end{array}$ & $\begin{array}{l}\text { Falso negativo para disección } \\
\text { aórtica debido a realce insufi- } \\
\text { ciente o enlentecido del lumen } \\
\text { falso aórtico }\end{array}$ \\
\hline & $\begin{array}{l}\text { Indicado incluso en pacientes } \\
\text { marginalmente estables }\end{array}$ & $\begin{array}{l}\text { Necesidad de TC controlado/ } \\
\text { gatillado por ECG, para investigar } \\
\text { la raíz aórtica y especialmente el } \\
\text { compromiso valvular }\end{array}$ & $\begin{array}{l}\text { Falso positivo para HIM en el } \\
\text { área de la raíz aórtica debido } \\
\text { a ateromatosis difusa u otras } \\
\text { enfermedades periaórticas }\end{array}$ \\
\hline & $\begin{array}{l}\text { Imagen de todas las estructuras } \\
\text { torácicas, buena definición de } \\
\text { lumen y pared aórtica }\end{array}$ & $\begin{array}{l}\text { Necesidad de traslado de paciente } \\
\text { de box de reanimación a unidad } \\
\text { de TC }\end{array}$ & $\begin{array}{l}\text { Diagnóstico falso positivo de } \\
\text { injuria aórtica/pseudoaneu- } \\
\text { risma traumático en caso de } \\
\text { remanente de ductus arterioso }\end{array}$ \\
\hline & $\begin{array}{l}\text { Detección de hematoma mural, } \\
\text { pericárdico o mediastínico }\end{array}$ & $\begin{array}{l}\text { En general no aporta información } \\
\text { respecto de la función valvular } \\
\text { aórtica o cardíaca }\end{array}$ & $\begin{array}{l}\text { Tejidos periaórticos tales como } \\
\text { fibrosis, inflamación y tumores, } \\
\text { como linfomas, pueden con- } \\
\text { fundir el diagnóstico }\end{array}$ \\
\hline & $\begin{array}{l}\text { Diferenciación entre lumen falso y } \\
\text { verdadero }\end{array}$ & & \\
\hline & $\begin{array}{l}\text { Detección confiable del desplaza- } \\
\text { miento de las calcificaciones de la } \\
\text { íntima }\end{array}$ & & \\
\hline
\end{tabular}


Tabla 5. Ventajas, desventajas y precaución de los distintos métodos imagenológicos para el diagnóstico del SAA ${ }^{17}$ (continuación)

\begin{tabular}{|c|c|c|c|}
\hline Examen & Ventajas & Desventajas & Precaución \\
\hline \multirow[t]{10}{*}{ RM } & $\begin{array}{l}\text { Muy alta sensibilidad y especi- } \\
\text { ficidad: ( } 95-98 \%) \text { en el caso de } \\
\text { disección aórtica aguda, y } 95 \% \\
\text { en HIM }\end{array}$ & $\begin{array}{l}\text { No puede realizarse en box de } \\
\text { reanimación }\end{array}$ & $\begin{array}{l}\text { Falsos positivos frente a } \\
\text { distorsión de las imágenes } \\
\text { por movimientos del paciente, } \\
\text { inhomogeneidad del campo } \\
\text { o en las interfases entre aire/ } \\
\text { pared aórtica }\end{array}$ \\
\hline & Sin radiación & $\begin{array}{l}\text { Intolerancia del paciente al exa- } \\
\text { men (claustrofobia) }\end{array}$ & \\
\hline & $\begin{array}{l}\text { Fácil detección del lumen falso y } \\
\text { verdadero }\end{array}$ & $\begin{array}{l}\text { Dificultades para monitorización } \\
\text { del paciente }\end{array}$ & \\
\hline & $\begin{array}{l}\text { El medio de contraste intravenoso } \\
\text { no es estrictamente necesario }\end{array}$ & $\begin{array}{l}\text { Contraindicación relativa en } \\
\text { pacientes usuarios de marcapasos } \\
\text { u otros dispositivos electromag- } \\
\text { néticos }\end{array}$ & \\
\hline & $\begin{array}{l}\text { Eficaz en la detección de enferme- } \\
\text { dades de la pared aórtica (HIM, } \\
\text { UAP) }\end{array}$ & $\begin{array}{l}\text { Contraindicado en pacientes he- } \\
\text { modinámicamente inestables }\end{array}$ & \\
\hline & $\begin{array}{l}\text { Distingue entre HIM y ateroma- } \\
\text { tosis no calcificada de la pared } \\
\text { aórtica }\end{array}$ & $\begin{array}{l}\text { Toma más tiempo que TC (15- } \\
20 \text { min) }\end{array}$ & \\
\hline & $\begin{array}{l}\text { Investigación simultánea de fun- } \\
\text { ción cardiaca, insuficiencia de la } \\
\text { válvula aórtica y enfermedades del } \\
\text { pericardio }\end{array}$ & $\begin{array}{l}\text { Difícil diagnóstico diferencial entre } \\
\text { HIM y otras enfermedades de la } \\
\text { pared aórtica, como ateromatosis, } \\
\text { tumores e inflamación }\end{array}$ & \\
\hline & $\begin{array}{l}\text { Gold standard para seguimien- } \\
\text { to de pacientes, operados y no } \\
\text { operados }\end{array}$ & $\begin{array}{l}\text { Necesidad de estudio controlado/ } \\
\text { gatillado por ECG cuando es nece- } \\
\text { sario estudiar raíz y válvula aórtica }\end{array}$ & \\
\hline & Ideal para pacientes en edad fértil & $\begin{array}{l}\text { Contraindicación relativa al uso de } \\
\text { medio de contraste con gadolinio } \\
\text { en pacientes con enfermedad } \\
\text { renal crónica o con antecedente } \\
\text { de reacción tipo alérgica previa }\end{array}$ & \\
\hline & & $\begin{array}{l}\text { Equipo clínico menos familiariza- } \\
\text { dos con la RM }\end{array}$ & \\
\hline \multirow[t]{5}{*}{ Aortografía } & $\begin{array}{l}\text { Sensibilidad y especificidad del } \\
88 \% \text { y } 94 \% \text { respectivamente para } \\
\text { el diagnóstico de la disección } \\
\text { aórtica aguda }\end{array}$ & $\begin{array}{l}\text { Exposición a radiación tanto para } \\
\text { el paciente como el operador; ma- } \\
\text { yores cantidades de radiación que } \\
\text { cualquier otro método de estudio } \\
\text { imagenológico }\end{array}$ & $\begin{array}{l}\text { Falso negativo en el diagnós- } \\
\text { tico de la disección aórtica } \\
\text { aguda si el falso lumen está } \\
\text { trombosado }\end{array}$ \\
\hline & $\begin{array}{l}\text { Excelente imagen de la patología } \\
\text { del lumen (DA, UAP) }\end{array}$ & $\begin{array}{l}\text { Necesidad de uso de medio de } \\
\text { contraste }\end{array}$ & $\begin{array}{l}\text { Falso negativo cuando no se } \\
\text { detecta el flap por inyección } \\
\text { proximal al foco de rotura } \\
\text { íntimal }\end{array}$ \\
\hline & Rol diagnóstico/terapéutico & $\begin{array}{l}\text { Tiempo de exploración prolongado } \\
\text { Pueden existir dificultades técnicas } \\
\text { con los accesos vasculares }\end{array}$ & $\begin{array}{l}\text { Posible falso negativo en el } \\
\text { diagnóstico de la UAP, salvo } \\
\text { exista adecuada desproyección }\end{array}$ \\
\hline & & $\begin{array}{l}\text { No puede diagnosticar HIM de la } \\
\text { pared aórtica }\end{array}$ & \\
\hline & & $\begin{array}{l}\text { Examen invasivo, requiere de un } \\
\text { laboratorio de hemodinamia y } \\
\text { médico intervencionista }\end{array}$ & \\
\hline
\end{tabular}

Tabla modificada a partir de E. Apostolakis et al. Rx: Radiografía; SAA: Síndrome aórtico agudo; TC: Tomografía computada; ECG: Electrocardiograma; TCMD: Tomografía computada multidetector; RM: Resonancia magnética; HIM: Hematoma intramural; UAP: Úlcera aórtica penetrante; DA: Disección aórtica. 


\section{Angio-TC}

- La angiografía por tomografía computarizada multicorte (TCMC) es el estudio de elección en pacientes hemodinámicamente estables $\mathrm{y}$ en los que se haya descartado derrame pleural o neumotórax masivo con radiografía o ecografía de tórax portátil obtenida en el box de atención, mientras se realiza la evaluación inicial.

- Técnica ampliamente disponible, tanto en hospitales públicos como privados, rápido de realizar y no necesita de gran colaboración por parte del paciente.

- Altamente sensible y específico en el SAA. Sensibilidad informada de $95-100 \%$ y una especificidad de $98-100 \%{ }^{18}$. La principal limitación de las imágenes es el movimiento de la raíz aórtica, donde la sensibilidad puede caer a menos de $80 \%{ }^{19}$. Para evitar este inconveniente el estudio se obtiene con sincronización electrocardiográfica, de manera de poder elegir las imágenes más apropiadas libres de distorsión por movimiento.

- Proporciona correlaciones anatómicas de gran precisión para la terapia quirúrgica o endovascular y aporta información para el seguimiento, a través de precisas reconstrucciones multiplanares y volumétricas.

- Su precisión ha mejorado sustancialmente con el desarrollo de la técnica Multidetector o Multicorte, TCMC) ${ }^{20}$. La TCMC permite amplia cobertura anatómica, mayor velocidad de adquisición y alta resolución.

- Debe incluirse una fase inicial sin contraste endovenoso para mejorar la detección del HIM o de colecciones hemáticas pericárdicas, mediastínicas o pleurales. El uso de contraste iodado endovenoso es escencial para el diagnóstico de estas condiciones. Cabe señalar que los contrastes iodados actuales han demostrado ser muy seguros, tanto desde el punto de vista del bajo riesgo de inducir reacciones anafilactoideas severas como en cuanto a inducir daño renal agudo, riesgos que en todo caso, la mayoría de las veces serán inferiores a dejar de hacer un diagnóstico de esta gravedad.

- El estudio debe abarcar la aorta completa, incluidos los vasos ilíacos y femorales. De esta manera, evaluar la extensión de la lesión y también mostrar si es que existe compromiso de las arterias viscerales.

\section{Técnica angioTCMC}

Es un examen trifásico, que debe abarcar desde los ápices pulmonares hasta los trocánteres femorales, de modo de incluir los vasos ilíacos y femorales. El ROI (región de interés) suele ser colocado sobre la aorta descendente (para minimizar artefactos de movimientos).

Se realiza una primera fase sin contraste, seguida de una fase arterial mediante técnica de bolus-tracking, utilizando $60-120 \mathrm{ml}$ de contraste iodado (370 mgl ml-1) a una velocidad de infusión de 3-6 ml/s según el peso del paciente, con el objetivo de lograr una opacificación del aorta de 250 $\mathrm{UH}$; la inyección de medio de contraste siempre debe ser seguida de un bolo de suero fisiológico a la misma velocidad de infusión, con el objetivo de conseguir una opacificación homogénea de la aorta y disminuir el artefacto.

La fase venosa se adquiere 1-2 min posterior a la inyección de contraste (permite ver un realce tardío del falso lumen y la alteración de la perfusión de órganos abdominales).

Para disminuir los artefactos de movimiento, el estudio debe ser gatillado según el ciclo cardíaco, sincronizando la adquisición de las imágenes con la díastole del ventrículo izquierdo mediante el uso del electrocardiograma.

Posteriormente, se utilizan técnicas de postproceso, como la reconstrucción multiplanar y volume rendering de modo de evaluar la aorta en toda su extensión y en caso de ser necesario, poder realizar una planificación quirúrgica óptima.

- Objetivos angioTCMC:

1. Confirmar diagnóstico.

2. Localizar origen de la rotura intimal.

3. Extensión y clasificación de la DA.

4. Compromiso de grandes vasos y sus ramas.

5. Identificación de indicadores de cirugía de emergencia. Ejemplo: hemorragia pericárdica, mediastínica y/o pleural.

- Desventajas angioTCMC:

1. Exposición a radiación. Esta desventaja toma relevancia en pacientes en edad fértil $y$ en el caso de uso indiscriminado, sin ajustarse a los criterios clínicos de sospecha (medicina defensiva).

2. Requiere contraste iodado endovenoso potencialmente nefrotóxico.

3. En general no es posible evaluar la insuficiencia de la válvula aórtica. 
Imágenes en síndrome aórtico agudo - C. Varela et al

\section{Manifestaciones imagenológicas de la DA, HIM y UAP en AngioTCMC}

\section{A. Disección aórtica}

- El primer paso es identificar dos lúmenes distintos (verdadero y falso), separados por el flap (Figura 6). En la mayoría de los casos, el lumen verdadero puede determinarse por su continuidad con un segmento no disecado de la aorta proximal o distal (Figura 7). El lumen falso generalmente es más grande que el verdadero ${ }^{21}$.

- La presencia de trombo intraluminal es un buen marcador de la luz falsa, pero en pacientes con un aneurisma degenerativo concomitante, el trombo puede estar presente en la luz verdadera.
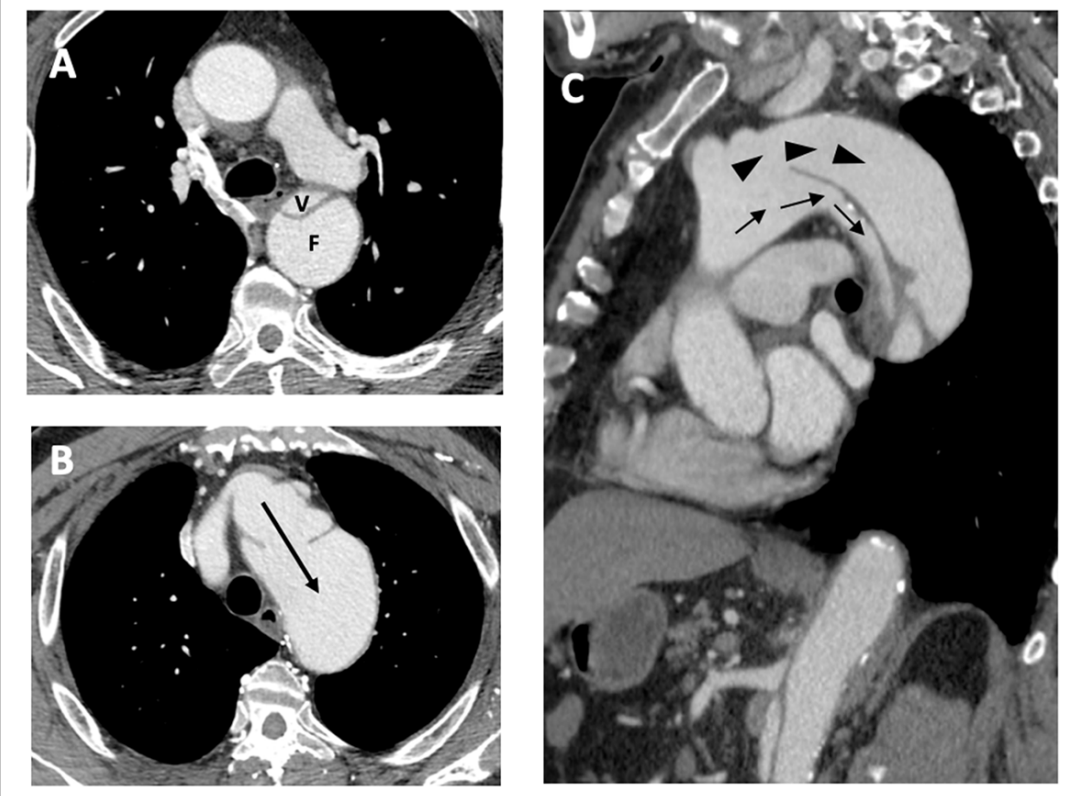

Figura 6. Signos de disección aórtica en TCMC. A. A nivel de aorta descendente se observa flap intimal que determina lumen verdadero $(\mathrm{V})$ y falso $(\mathrm{F})$. B. En corte axial más craneal al anterior, se observa sitio de entrada de la disección desde el lumen verdadero al lumen falso (Flecha). C. A nivel de aorta descendente proximal, en reconstrucción sagital, se identifica foco de entrada a lumen falso (Cabezas de flechas) de mayor tamaño, y lumen verdadero de menor tamaño (Flechas).

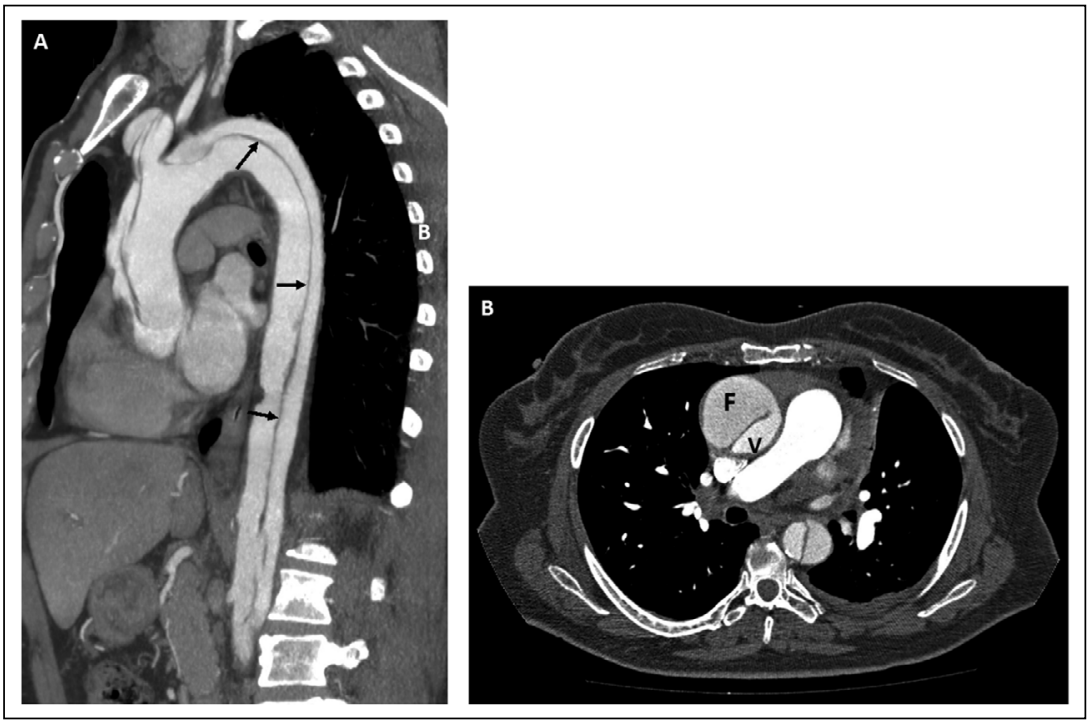

Figura 7. Signos de disección aórtica tipo A en TCMC. A. Se observa flap de disección que compromete todos los segmentos de la aorta torácica, tipo A de Stanford (Flechas negras). B. A nivel de aorta ascendente se aprecia doble lumen, uno verdadero $(\mathrm{V})$ y uno falso de mayor tamaño (F). 
- Un lumen verdadero comprimido es un hallazgo clave, que eleva sustancialmente el índice de sospecha de un síndrome de mala perfusión renal, visceral y/o de las extremidades inferiores.

- La curvatura del colgajo de disección hacia la luz verdadera se observa en $63 \%$ de las disecciones de tipo B agudas, pero solo en $25 \%$ de las disecciones crónicas ${ }^{21}$.

- Hallazgos secundarios incluyen desplazamiento interno de calcificaciones intimales (Figura 8) o una íntima hiperdensa, captación tardía del lumen falso, ensanchamiento aórtico, y aparición de hematoma mediastínico, pleural o pericárdico ${ }^{22}$.

- Las complicaciones de la DA tipo A son: hemopericardio, que puede conducir a taponamiento cardíaco, disección de la válvula aórtica y compromiso del ostium de las arterias coronarias.

- Las complicaciones de la DA tipo B, pueden dividirse en dos grupos:

- Ruptura franca, con aparición de derrame pleural denso o compromiso hemodinámico por hemorragia.

- De tipo isquémico, en órganos o extremidades $^{23}$ (Figura 9).
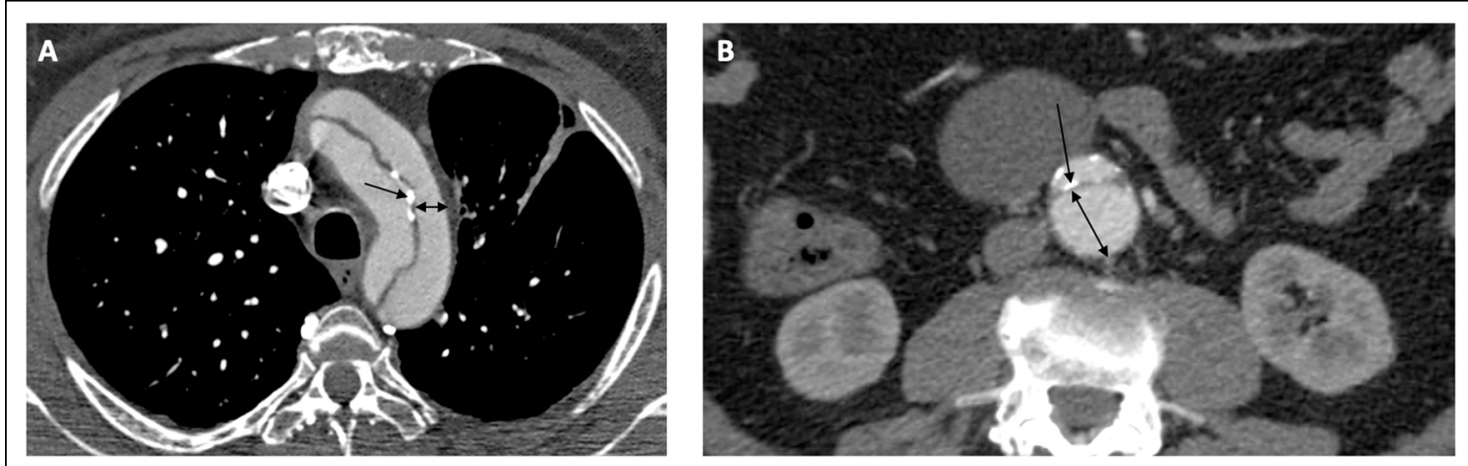

Figura 8. Signo del desplazamiento de calcificaciones parietales: DA en TCMC. A. A nivel del arco aórtico se identifica un flap de disección con calcificaciones intimales (Flecha) las que han sido desplazadas por el flujo a través del lumen falso (Doble Flecha). B. En corte axial de aorta abdominal, se vuelve a observar un flap de disección con calcificaciones intimales (Flecha) desplazadas desde pared posterior por flujo a través del lumen falso (doble flecha).
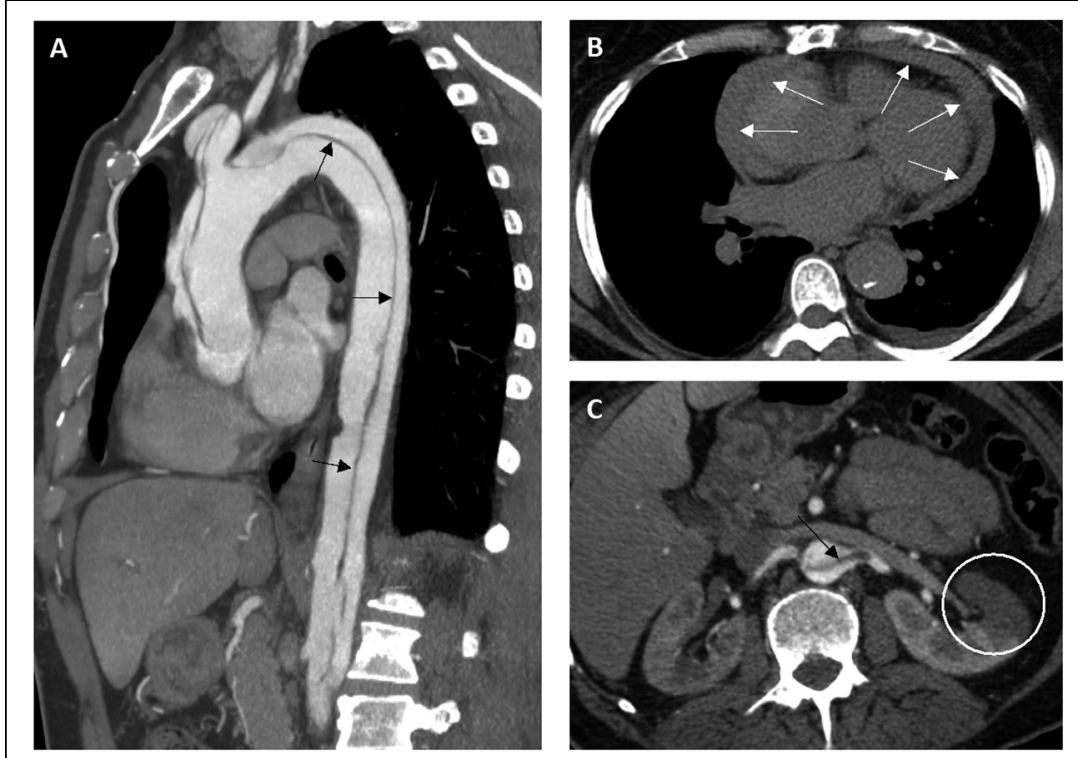

Figura 9. Signos de complicaciones de la DA tipo A. A. Reconstrucción sagital donde se observa extensión del flap intimal desde la raíz aórtica hasta la aorta abdominal (Flechas negras). B. Corte axial en fase sin contraste, muestra derrame pericárdico hiperdenso compatible con hemopericardio (Flechas blancas). C. Corte axial a nivel de hilios renales, que muestra extensión del flap de disección desde la aorta hacia la arteria renal izquierda (Flecha), asociada a imagen hipodensa de bordes netos compatible con infarto renal segmentario de territorio vascular anterior (Círculo blanco). 


\section{B. Hematoma intramural22,24 (Figura 10)}

- En TCMC sin contraste se ve una semiluna hiperdensa en la pared de la aorta o arterias comprometidas, pudiendo haber desplazamiento de las calcificaciones intimales.

- Por otro lado, en TCMC con contraste no se evidencia realce de HIM.

- Es importante señalar que la imagen de un HIM y una DA con trombosis completa del lumen falso es muy similar y puede ser difícil de diferenciar. El desplazamiento de calcificaciones intimales sugiere DA.

\section{C. Úlcera aórtica penetrante ${ }^{22}$ (Figura 11)}

- En TCMC con contraste se ve una imagen diverticular que se extiende más allá del contorno aórtico.

- Se puede observar una úlcera focal con un hematoma subintimal adyacente.

- Se puede diferenciar de una DA por asociarse a:

- Enfermedad aterosclerótica extensa y dilatación luminal.

- Ausencia de doble lumen aórtico.

\section{Otros métodos de imagen}

\section{Ecocardiografía}

Segundo examen de imagen más utilizado en el diagnóstico inicial de SAA. Es especialmente útil en pacientes hemodinámicamente inestables, ya que puede realizarse en el box de reanimación. Puede identificar regurgitación de la válvula aórtica, derrame pericárdico hemorrágico, taponamiento y anormalidades regionales en el movimiento de la pared. Puede ser realizado de dos maneras:

- Transesofágico (ETE): estudio inicial en pacientes hemodinámicamente inestables. Tiene una sensibilidad de $98 \%$ y una especificidad de $63-96 \% \%^{25,26}$. Dentro de sus ventajas cabe mencionar que puede realizarse en el box de reanimación y produce un diagnóstico en minutos. Por el contrario, tiene como desventajas que requiere intubación esofágica, por lo tanto, sedación, pudiendo tener efectos adversos en pacientes lábiles. Además, requiere operadores experimentados (médicos y técnicos) para garantizar resultados precisos.

- Transtorácico (ETT): Alternativa menos utilizada que el ETE. Puede identificar rápidamente la DA proximal ${ }^{26}$ y también sus complicaciones como insuficiencia aórtica, taponamiento cardíaco, disfunción sistólica. Su principal desventaja es que no permite ver adecuadamente el resto de la aorta torácica (ascendente media y distal, cayado y la aorta descendente), ni la presencia de otras complicaciones distales a su campo de visión. Su mayor ventaja es que se puede realizar de forma portátil al lado de
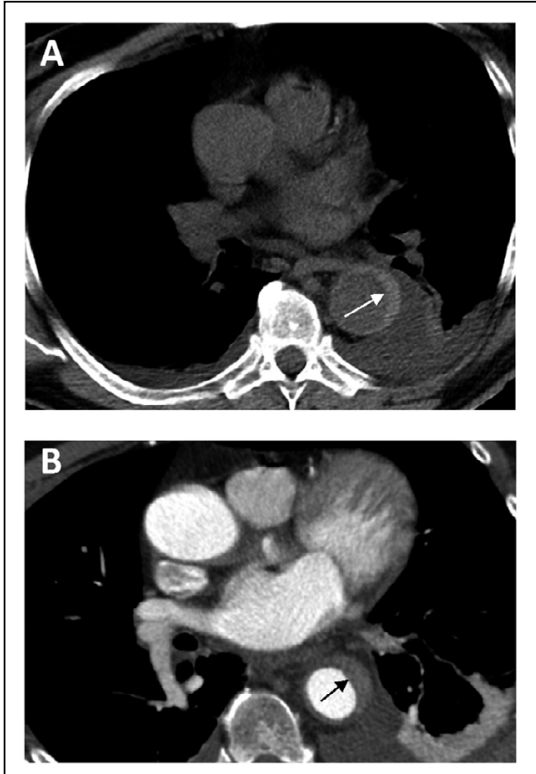

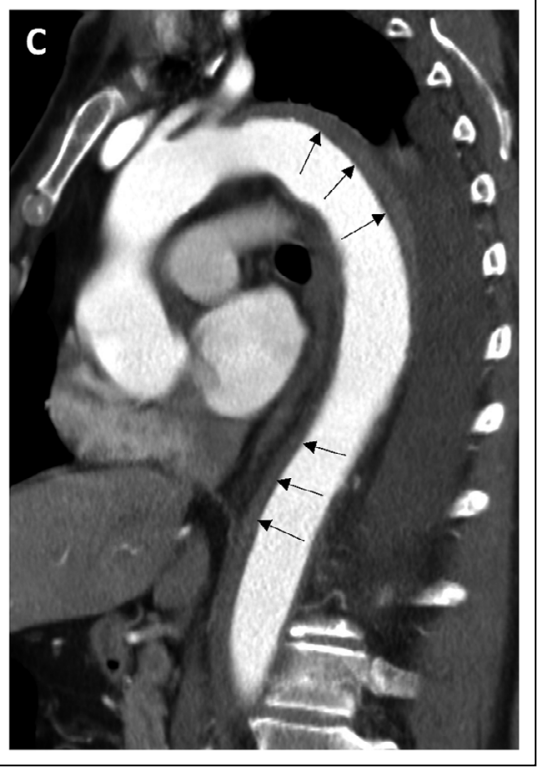

Figura 10. Signos de hematoma intramural en TCMC. A. Corte axial sin contraste endovenoso. En aorta descendente se observa imagen de semiluna hiperdensa en la pared de la aorta descendente (Flecha blanca), compatible con HIM. B. Fase con contraste, que muestra ausencia de doble lumen (Flecha negra) que respalda el diagnóstico de HIM. C. Reconstrucción sagital, donde se observa HIM que se extiende a través de la aorta torácica descendente mostrando una característica distribución espiroídea, ya que posee afectación parcial de la pared aórtica. 

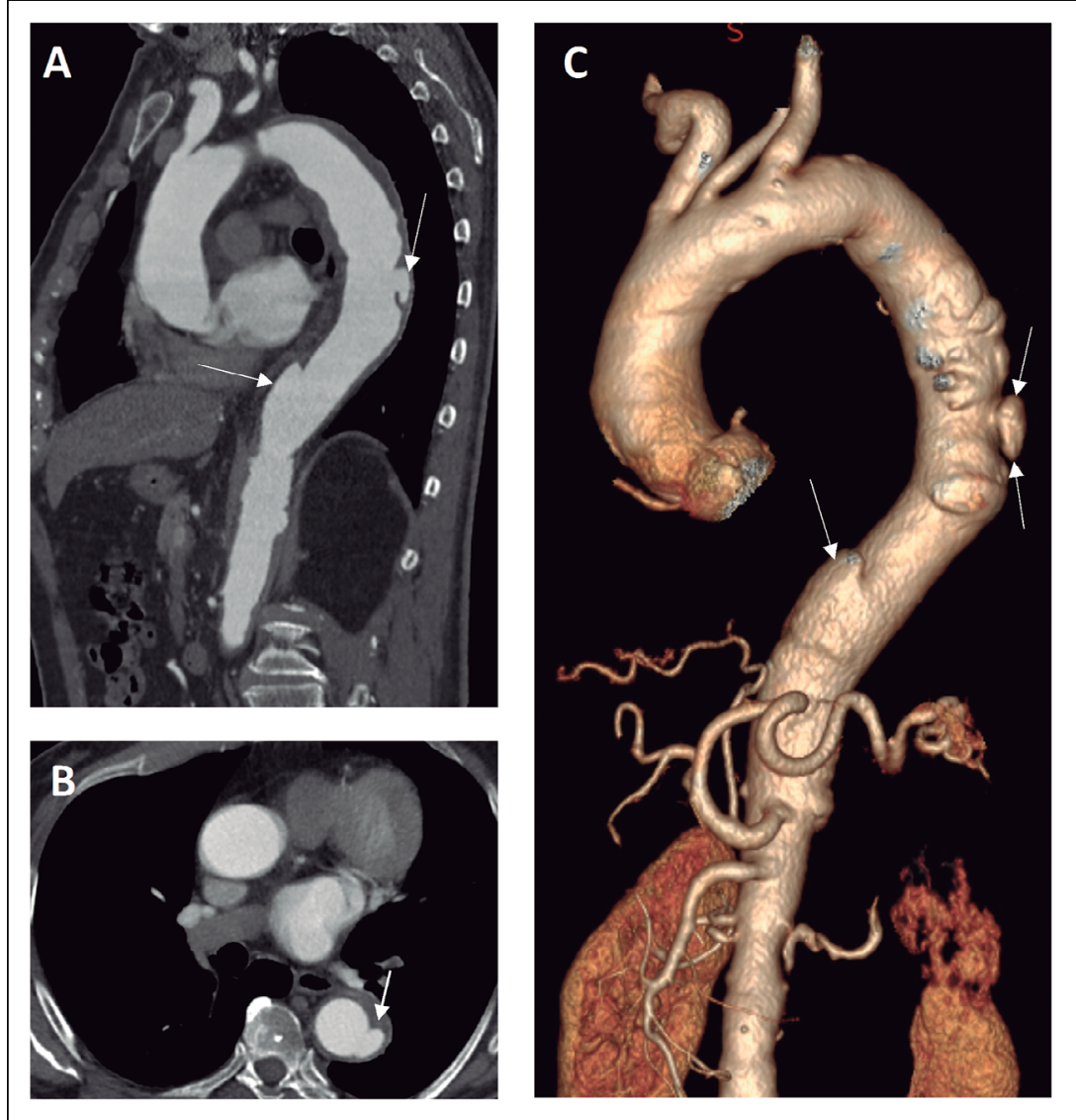

Figura 11. Ulcera Aórtica Penetrante en TCMC. A. Reconstrucción sagital en fase con contraste, donde se observa engrosamiento de la pared aórtica secundaria a extensa ateromatosis. En este contexto se identifican dos salientes de perfil compatibles con UAP (Flechas blancas). B. Corte axial en fase con contraste, muestra UAP de aorta descendente media, como una colección de contraste que se extiende hacia planos parietales profundos (Flecha blanca). C. Reconstrucción volumétrica, se identifican las UAP como salientes del perfil aórtico (Flechas blancas). la cama del paciente. También se debe considerar que su sensibilidad y especificidad son inferiores al TCMC, RM y ETE.

\section{Radiografía de tórax (Figura 12)}

- Su utilidad es descartar otras causas de dolor torácico como neumotórax o derrame pleural masivo $^{11}$.

- Signos asociados a SAA: Ensanchamiento del mediastino $(60-90 \%)^{11,27}$, ensanchamiento y mala definición del contorno aórtico, desplazamiento de la pared aórtica calcificada, opacificación de la ventana aorticopulmonar y derrame pleural (19\%).

\section{Resonancia Magnética (RM)}

- Es una alternativa al angioTCMC, en pacientes hemodinámicamente estables y según la disponibilidad local de la técnica. La angiografía por RM con Gadolinio, tiene una excelente sensibilidad y especificidad de $95-100 \%$ para diagnosticar $\mathrm{DA}^{28}$. Puede evaluar flujo diferencial entre los lúmenes verdadero y falso y detectar trombosis en el lumen falso.

- Rara vez se utiliza, por la mayor disponibilidad de la TCMC en los Servicios de Urgencia. Es una herramienta de diagnóstico altamente precisa para la detección de DA. Por sus desventajas, se usa como segunda línea diagnóstica, cuando el estudio imagenológico inicial es inadecuado ${ }^{29}$. La RM puede demostrar con gran especificidad el contenido hemático parietal en el HIM. Si no es posible usar contraste, se puede detectar el lumen falso, con secuencias sensibles al flujo. También permite cuantificar insuficiencia valvular aórtica, y también es la modalidad de elección para estudios de seguimiento a largo plazo, sobre todo en pacientes jóvenes, con disección tipo B. 

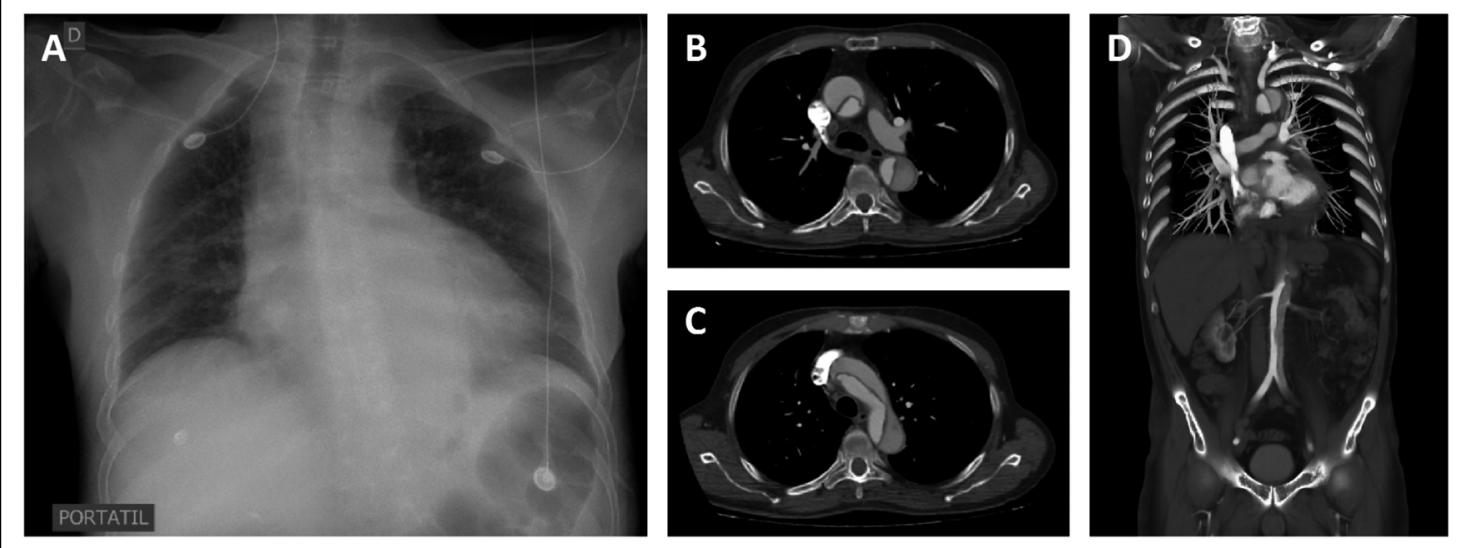

Figura 12. A. Radiografía de tórax portátil que descarta neumonía, neumotórax o derrame masivo. B, C y D. TCMC cortes axiales y reconstrucción coronal que muestran DA tipo A que se extiende hasta segmento infrarrenal.

- Dentro de sus ventajas, es muy importante que evita el exceso de exposición a la radiación y que los agentes de contraste basados en gadolinio no son nefrotóxicos en las dosis habituales.

- En cuanto a sus desventajas: los pacientes deben colaborar permaneciendo inmóviles durante más de 30 minutos. No se puede realizar en pacientes con claustrofobia. Existe contraindicación relativa en portadores de marcapasos, clips de aneurisma y otros dispositivos electromagnéticos. Su disponibilidad es limitada, existe poca experiencia y su análisis es lento. Además, hay inquietudes sobre la monitorización y la relativa inaccesibilidad al paciente durante la exploración prolongada. Y, por último, se debe evitar la administración de contraste basado en gadolinio en pacientes con enfermedad renal crónica (sobre todo en dializados) por el riesgo de desarrollar una fibrosis sistémica nefrogénica.

\section{Arteriografía por catéter (aortografía)}

- Antiguo gold standard. Se usaba en pacientes con sospecha de DA ascendente, en quienes las imágenes no invasivas no eran concluyentes. Es solo moderadamente sensible para el diagnóstico de $\mathrm{DA}^{5}$.

- Hallazgos compatibles con DA: Distorsión de la columna de contraste normal, reversión del flujo o estasis en un canal falso, falla en el llenado de las ramas principales e insuficiencia de la válvula aórtica.
- Posee rol terapéutico en DA del segmento descendente donde es posible instalar endoprótesis cubierta para excluir el lumen falso ${ }^{30}$.

\section{Conclusión}

Las imágenes son fundamentales para el diagnóstico del SAA, y el AngioTC de tórax es el examen de primera elección. Su gran rendimiento diagnóstico, basado en la exquisita demostración anatómica de las lesiones de la pared aórtica, se incrementa con su rapidez de ejecución y su amplia disponibilidad. Las distintas entidades que conforman este síndrome pueden ser diferenciadas a través de sus manifestaciones en TC, determinando además su extensión y sus probables complicaciones. Para utilizar apropiadamente esta extraordinaria herramienta se debe aplicar un alto nivel de sospecha clínica, tamizando a los pacientes de acuerdo a sus factores de riesgo. El diagnóstico precoz es fundamental para mejorar la sobrevida de los pacientes.

\section{Referencias}

1. Latarjet M, Ruiz Liard A, Editores. Aorta. Anatomía Humana. Buenos Aires, Argentina: Editorial Médica Panamericana; 2005. p. 979-80.

2. Manning W, Black J. Overview of acute aortic dissection and other acute aortic syndromes. March, 2018. Obtained from UpToDate. 
3. Nienaber C, Eagle K. Aortic Dissection: New Frontiers in Diagnosis and Management: Part I: from etiology to diagnostic strategies. Circulation 2003; 108 (5): 62835.

4. Maraj R, Rerkpattanapipat P, Jacobs L, Makornwattana P, Kotler M. Meta-analysis of 143 reported cases of aortic intramural hematoma. Am J Cardiol 2000; 86 (6): 664-8.

5. Rizzo R, Aranki S, Aklog L, Couper G, Adams D, Collins J Jr, et al. Rapid noninvasive diagnosis and surgical repair of acute ascending aortic dissection. Improved survival with less angiography. J Thorac Cardiovasc Surg 1994; 108 (3): 567-74.

6. Hayashi H, Matsuoka Y, Sakamoto I, Sueyoshi E, Okimoto T, Hayashi K, et al. Penetrating atherosclerotic ulcer of the aorta: imaging features and disease concept. Radiographics 2000; 20 (4): 995-1005.

7. Svensson L, Labib S, Eisenhauer A, Butterly J. Intimal tear without hematoma: an important variant of aortic dissection that can elude current imaging techniques. Circulation 1999; 99 (10): 1331-6.

8. Mukherjee D, Evangelista A, Nienaber C, Sechtem U, Suzuki T, Trimachi S et al. Implications of periaortic hematoma in patients with acute aortic dissection (from the International Registry of Acute Aortic Dissection). Am J Cardiol 2005; 96 (12): 1734-8.

9. Pape L, Awais M, Woznicki E, Suzuki T, Trimarchi S, Evangelista A, et al. Presentation, Diagnosis, and Outcomes of Acute Aortic Dissection: 17-Year Trends From the International Registry of Acute Aortic Dissection. J Am Coll Cardiol 2015; 66 (4): 350-8.

10. Mertens R, Arriagada I, Valdés F, Krämer A, Mariné L, Bergoeing M, et al. Tratamiento endovascular de la disección aórtica tipo B mediante endoprótesis. Rev Med Chile 2008; 136 (11): 1431-8.

11. Hagan $P$, Nienaber $C$, Isselbacher E, Bruckman D, Karavite $\mathrm{D}$, Evangelista $\mathrm{A}$, et al. The International Registry of Acute Aortic Dissection (IRAD): new insights into an old disease. JAMA 2000; 283 (7): 897-903.

12. Nazerian P, Mueller C, Soeiro A, Leidel B, Salvadeo $S$, Giachino F, et al. Diagnostic Accuracy of the Aortic Dissection Detection Risk Score Plus D-Dimer for Acute Aortic Syndromes: The ADvISED Prospective Multicenter Study. Circulation 2018;137 (3): 250-8.

13. Black J, Manning W. Clinical features and diagnosis of acute aortic dissection. February 2018. Obtained from UpToDate.

14. Vardhanabhuti V, Nicol E, Morgan-Hughes G, Roobottom C, Roditi G, Hamilton M, et al. Recommendations for accurate CT diagnosis of suspected acute aortic syndrome (AAS)-on behalf of the British Society of
Cardiovascular Imaging (BSCI)/British Society of Cardiovascular CT (BSCCT). Br J Radiol 2016; 89 (1061): 20150705.

15. Martínez F, Avendaño G, Brinkmann M, Cortés C, Carrillo I. Traslado de pacientes críticos. Rev Hosp Clín Univ Chile 2013; 25: 246-52.

16. Knight PH, Maheshwari N, Hussain J, Scholl M, Hughes M, Papadimos TJ, et al. Complications during intrahospital transport of critically ill patients: Focus on risk identification and prevention. Int J Crit Illn Inj Sci 2015; 5: 256-64.

17. Apostolakis E, Papakonstantinou N, Baikoussis N, Petrou A, Goudevenos J. Imaging of acute aortic syndrome: advantages, disadvantages and pitfalls. Hellenic J Cardiol 2015;56 (2): 169-80.

18. Willoteaux S, Nedelcu C, Bouvier A, Hoareau J, Biere L, Thouveny F, et al. [Acute aortic syndrome: which type of imaging to use?]. Presse Med 2011; 40 (1 Pt 1): 43-53.

19. Khan I, Nair C. Clinical, diagnostic, and management perspectives of aortic dissection. Chest 2002; 122 (1): 311-28.

20. Hayter R, Rhea J, Small A, Tafazoli F, Novelline R. Suspected aortic dissection and other aortic disorders: multi-detector row CT in 373 cases in the emergency setting. Radiology 2006; 238 (3): 841-52.

21. LePage M, Quint L, Sonnad S, Deeb G, Williams D. Aortic dissection: CT features that distinguish true lumen from false lumen. AJR Am J Roentgenol 2001; 177 (1): 207-11.

22. Sebastià C, Pallisa E, Quiroga S, Álvarez-Castells A, Domínguez R, Evangelista A. Aortic dissection: diagnosis and follow-up with helical CT. Radiographics 1999; 19 (1): 45-60.

23. Vega J, Zamorano J, Pereira N, Galleguillos A. Síndrome aórtico agudo. Revisión de la literatura y actualización del tema. Rev Med Chile 2014; 142 (3): 344-52.

24. Gutschow S, Walker C, Martínez-Jiménez S, Rosado-de-Christenson M, Stowell J, Kunin J. Emerging Concepts in Intramural Hematoma Imaging. RadioGraphics 2016; 36 (3): 660-74.

25. Moore A, Eagle K, Bruckman D, Moon BS, Malouf JF, Fattori R, et al. Choice of computed tomography, transesophageal echocardiography, magnetic resonance imaging, and aortography in acute aortic dissection: International Registry of Acute Aortic Dissection (IRAD). Am J Cardiol 2002; 89 (10): 1235-8.

26. Nienaber C, von Kodolitsch Y, Nicolas V, Siglow V, Piepho A, Brockhoff C, et al. The diagnosis of thoracic aortic dissection by noninvasive imaging procedures. $\mathrm{N}$ Engl J Med 1993; 328 (1): 1-9.

27. Von Kodolitsch Y, Nienaber C, Dieckmann C, Schwartz 
A, Hofmann T, Brekenfeld C. Chest radiography for the diagnosis of acute aortic syndrome. Am J Med 2004; 116 (2): 73-7.

28. Gebker R, Gomaa O, Schnackenburg B, Rebakowski J, Fleck E, Nagel E. Comparison of different MRI techniques for the assessment of thoracic aortic pathology: 3D contrast enhanced MR angiography, turbo spin echo and balanced steady state free precession. Int J Cardio- vasc Imaging 2007; 23 (6): 747-56.

29. Braverman A. Aortic dissection: prompt diagnosis and emergency treatment are critical. Cleve Clin J Med 2011; 78 (10): 685-96.

30. Cigarroa J, Isselbacher E, DeSanctis R, Eagle K. Diagnostic imaging in the evaluation of suspected aortic dissection. Old standards and new directions. N Engl J Med 1993; 328 (1): 35-43. 